\title{
Imaging for the Diagnosis and Management of Traumatic Brain Injury
}

\author{
Jane J. Kim and Alisa D. Gean \\ Department of Radiology, San Francisco General Hospital, University of California, 1001 Potrero Avenue, Box 1325 San Francisco, \\ California 94143
}

\begin{abstract}
Summary: To understand the role of imaging in traumatic brain injury (TBI), it is important to appreciate that TBI encompasses a heterogeneous group of intracranial injuries and includes both insults at the time of impact and a deleterious secondary cascade of insults that require optimal medical and surgical management. Initial imaging identifies the acute primary insult that is essential to diagnosing TBI, but serial imaging surveillance is also critical to identifying secondary injuries such as cerebral herniation and swelling that guide neurocritical management. Computed tomography (CT) is the
\end{abstract}

mainstay of TBI imaging in the acute setting, but magnetic resonance tomography (MRI) has better diagnostic sensitivity for nonhemorrhagic contusions and shear-strain injuries. Both CT and MRI can be used to prognosticate clinical outcome, and there is particular interest in advanced applications of both techniques that may greatly improve the sensitivity of conventional CT and MRI for both the diagnosis and prognosis of TBI. Key Words: TBI, hemorrhage, subdural, epidural, contusion, traumatic shear injury.

\section{INTRODUCTION}

Traumatic brain injury (TBI) is a significant cause of morbidity and mortality worldwide, with substantial associated economic costs to the healthcare system. It is estimated that 10 million people sustain TBI each year worldwide, and the Centers for Disease Control and Prevention in the USA estimates that 1.7 million people suffer TBI annually [1]. The cost of TBI in the USA, including direct medical costs and indirect costs of lost productivity, is estimated to total $>60$ billion dollars annually [1]. Over the long term, TBI patients also suffer functional and cognitive changes and develop medical conditions, such as epilepsy, that require long-term or lifelong supportive and medical care.

The primary causes of TBI vary according to patient age. Falls are the leading cause of TBI in children up to 4 years of age and elderly individuals $>75$ years of age. Among adolescents, motor-vehicle accidents are the chief cause of TBI. Sports and recreation-related injuries,

Electronic supplementary material The online version of this article (doi:10.1007/s13311-010-0003-3) contains supplementary material, which is available to authorized users.

\footnotetext{
Address correspondence and reprint requests to: Jane J. Kim Department of Radiology, San Francisco General Hospital, University of California, 1001 Potrero Avenue, Box 1325 San Francisco, CA 94143. E-mail: jane.kim@radiology.ucsf.edu
}

assaults and firearm use, and blast injuries for active duty military personnel are other common causes of TBI.

\section{PATHOPHYSIOLOGY OF TBI}

The damage to the brain from TBI is traditionally divided into primary and secondary injuries. Primary injuries, such as hematomas and traumatic axonal injury (TAI), occur as a direct result of the traumatic impact. Secondary injuries develop minutes to days after the primary trauma and include a complex biochemical cascade of events leading to cerebral swelling and herniation that is triggered by the primary traumatic event. This classification highlights that TBI is not a onetime event but rather a continuous and progressive injury that necessitates optimal medical and surgical management of cerebral oxygenation, intracranial pressure (ICP) and cerebral perfusion pressure (CPP) to maximize patient recovery and prevent successive injury.

Primary traumatic injuries, such as intracranial hemorrhage, can trigger secondary insults by raising the ICP. CPP (i.e., the pressure gradient driving oxygen and nutrient delivery to the brain) is the difference between mean arterial pressure and ICP. CPP is used as an index of cerebral blood flow. When the ICP increases due to TBI (or systemic blood pressure falls), the CPP will fall, and the brain will become ischemic unless intact cerebral 
autoregulation induces compensatory cerebrovascular vasodilatation to maintain adequate blood flow to the brain. However, normal cerebral autoregulation is frequently disrupted in patients with TBI, particularly in young patients. Also, at low CPP values (below a threshold of $\sim 50-60 \mathrm{~mm} \mathrm{Hg}$ ), autoregulation seems to be impaired, and the brain is likely to become ischemic $[2,3]$. With ischemia and the resultant decreased oxygen and glucose delivery to the brain, complex biochemical and cellular pathways are triggered that further aggravate injury to the brain. Excitatory neurotransmitters (primarily glutamate) are released into the tissue that initiate various pathophysiologic processes, including excessive calcium influx into cells, resulting in mitochondrial dysfunction, cellular swelling, free radical production, and eventual neuronal death.

\section{PRINCIPLES OF MANAGEMENT OF TBI}

Current management of TBI in the neurointensive care unit is focused on preventing or mitigating the abovementioned cascade of secondary injuries by maintaining adequate perfusion to the brain. This approach requires vigilant neuromonitoring and control of blood pressure and oxygenation to avoid both hypotension and hypoxemia, which can increase morbidity and mortality [4-6]. TBI patients in whom autoregulation is severely impaired depend entirely on blood pressure to maintain cerebral blood flow to the brain, a condition termed "pressurepassive" flow. Adequate blood pressure support is therefore critical.

ICP is also monitored in all patients with severe TBI as defined by a Glasgow Coma Scale (GCS) score of 3-8, and an abnormal computed tomography (CT) scan [7, 8]. ICP is conventionally measured by an external ventricular drainage catheter, though parenchymal, subarachnoid, subdural, and epidural devices can also be used. As increased ICP may portend cerebral herniation or potentially compromised CPP and is associated with a worse prognosis, rigorous measures to lower the ICP are used. These includes both medical treatment with hyperosmolar therapy, cautious hyperventilation, and appropriate sedation/ analgesia, as well as surgical treatment with cerebrospinal fluid (CSF) drainage via the external ventricular drainage catheter, intracranial hematoma evacuation and, if necessary, decompressive craniectomy [9-11].

Finally, ensuring that CPP is maintained at an adequate level to avoid ischemic injury is important; again, threshold values for CPP are $\sim 50-60 \mathrm{~mm} \mathrm{Hg}$, as cerebral ischemia may occur below this range $[2,3]$.

A large number of neuroprotective agents that modulate the deleterious biochemical cascade triggered by cerebral ischemia to further compound injury have been studied. Numerous neuroprotective strategies, including the use of agents to antagonize excessive excitotoxic glutamate activity, block voltage-gated calcium channels and intracellular calcium influx, reduce mitochondrial dysfunction, and limit free radical damage, have been evaluated in both experimental animal models and clinical trials [12-14]. Unfortunately, none of these agents have shown convincing benefit in randomized control trials to date. This lack of an efficacious outcome may reflect a number of factors, including the heterogeneity of TBI patients in these trials and the difficulty in knowing whether the pathophysiologic mechanisms targeted in these patients are indeed active at the time of drug delivery.

\section{ROLE OF IMAGING IN DIAGNOSIS AND MANAGEMENT OF TBI}

Imaging is critical to both the diagnosis and management of TBI. For diagnosis of TBI in the acute setting, noncontrast $\mathrm{CT}$ is the modality of choice as it quickly and accurately identifies intracranial hemorrhage that warrants neurosurgical evacuation. CT readily identifies both extra-axial hemorrhage (epidural, subdural, and subarachnoid/intraventricular hemorrhage) and intraaxial hemorrhage (cortical contusion, intraparenchymal hematoma, and TAI or shear injury). While CT is the mainstay of TBI imaging in the acute setting, magnetic resonance imaging (MRI) has better diagnostic sensitivity for certain types of injuries that are not necessarily hemorrhagic, including cortical contusions and nonhemorrhagic traumatic axonal injuries.

For the management of TBI patients, noncontrast CT readily identifies the progression of hemorrhage and signs of secondary injury relevant to neurocritical care, such as cerebral swelling, herniation, and hydrocephalus.

In this review, we discuss each type of primary and secondary brain injury in turn, with an emphasis on the imaging features that are of particular relevance to medical and surgical management and decision-making.

\section{PRIMARY INJURIES IN TBI PATIENTS}

\section{Epidural hematoma}

An epidural hematoma (EDH) typically occurs at the site of traumatic impact or "coup" site and is almost always associated with an overlying skull fracture [15]. Injury to a meningeal artery/vein, diploic vein, or dural venous sinus results in a classically lentiform-shaped collection of blood that strips the dura away from the inner table of the skull. EDHs are most common in the temporal or temporoparietal regions, often due to laceration of the middle meningeal artery or one of its branches (FIG. 1) [16]. A venous EDH can occur in the 

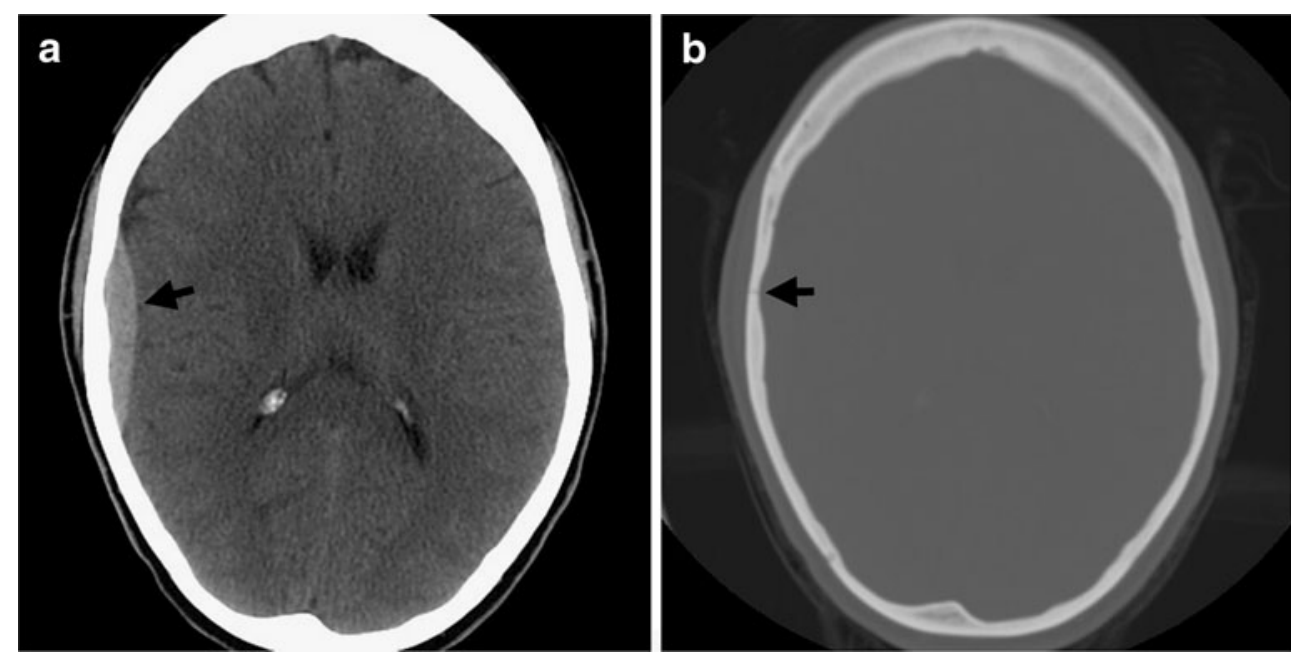

FIG. 1. Classic arterial epidural hematoma. (a) Axial noncontrast CT in brain windows shows a lentiform, high attenuation collection (arrow) adjacent to the right temporal lobe, consistent with an EDH caused by injury to a branch of the middle meningeal artery. (b) The skull fracture that is almost invariably seen with an EDH is best appreciated on bone windows (arrow). CT = computed tomography; $\mathrm{EDH}=$ epidural hematoma. (High resolution version of this image is available in the electronic supplementary material.)

posterior fossa due to laceration of the transverse/ sigmoid sinus, or in the middle cranial fossa due to injury of the sphenoparietal sinus (FIG. 2) [17].

On imaging, EDHs typically appear lentiform or biconvex in shape and do not cross cranial sutures because the periosteal layer of the dura adheres tightly to the suture. However, unlike subdural hematomas, EDHs can cross the midline where the periosteal layer of the dura forms the outer wall of the superior sagittal sinus, which may be displaced from the inner table of the skull (FIG. 3). Also, unlike subdural hematomas, EDHs can extend above and below the tentorium cerebelli, which is a dural reflection separating the cerebellum from the occipital lobes.

The decision to surgically evacuate an acute EDH is based on a number of findings, including the patient's GCS score, pupil exam, comorbidities, age, and CT findings. On imaging, findings that suggest a poorer prognosis and prompt early surgical evacuation include significant hematoma thickness $(>15 \mathrm{~mm})$, large hematoma volume $\left(>30 \mathrm{~cm}^{3}\right)$, significant midline shift ( $>5 \mathrm{~mm}$ ), compression of the basilar cisterns, and mixed density of the hematoma, which often indicates active bleeding (Table 1) [16, 18-20]. The hematoma volume can be calculated by the $A B C / 2$ method described by Kothari et al [21]. where the CT slice demonstrating the largest area of hemorrhage is selected, and $A$ reflects the diameter of the hemorrhage on that slice, $B$ is the measurement taken perpendicular to $A$, and $C$ is the approximate number of $10-\mathrm{mm}$ slices with hemorrhage.

\section{Subdural hematoma}

A subdural hematoma (SDH) can occur either at the coup or contrecoup site, although the latter is more common. Injury to superficial bridging veins results in bleeding between the meningeal layer of the dura and arachnoid, and blood may continue to accumulate in this space as bridging veins are progressively stretched and injured. SDHs commonly occur over the cerebral convexities, along the tentorium cerebelli, and along the falx cerebri, in descending order of frequency [22].

On imaging, SDHs appear crescentic in shape and do not cross the midline; they can, however, cross cranial suture lines (unlike EDHs). Small SDHs can be extremely subtle, so careful adjustment of window settings and scrutiny of the falx cerebri and tentorium cerebelli for subtle asymmetric high density are important (FIG. 4). Most SDHs in the setting of acute TBI are homogeneously hyperintense on noncontrast CT scans. However, isodense hematomas can occur if the patient is severely anemic or CSF from an arachnoid tear is admixed with hemorrhage. Heterogeneous SDHs can occur in the context of active bleeding, coagulopathy, or acute-on-chronic hemorrhage (FIG. 5).

Surgical management of SDHs, like EDHs, depends on a number of factors, including the patient's GCS score, pupil exam, comorbidities, age, and CT findings. Neurologic deterioration over time is another important factor in surgical decision-making [23]. As with EDHs, CT findings that appear to predict a poor prognosis include clot thickness, midline shift, and basilar cistern compression. While specific threshold values are difficult to determine, and vary among different studies, the Surgical Management of Traumatic Brain Injury Guidelines issued by a consortium of neurosurgeons following a review of the available scientific literature recommends surgical evacuation of acute SDHs that are $>10 \mathrm{~mm}$ thick or those causing a midline shift $>5 \mathrm{~mm}$ visible on the CT scan (Table 2) [23]. 

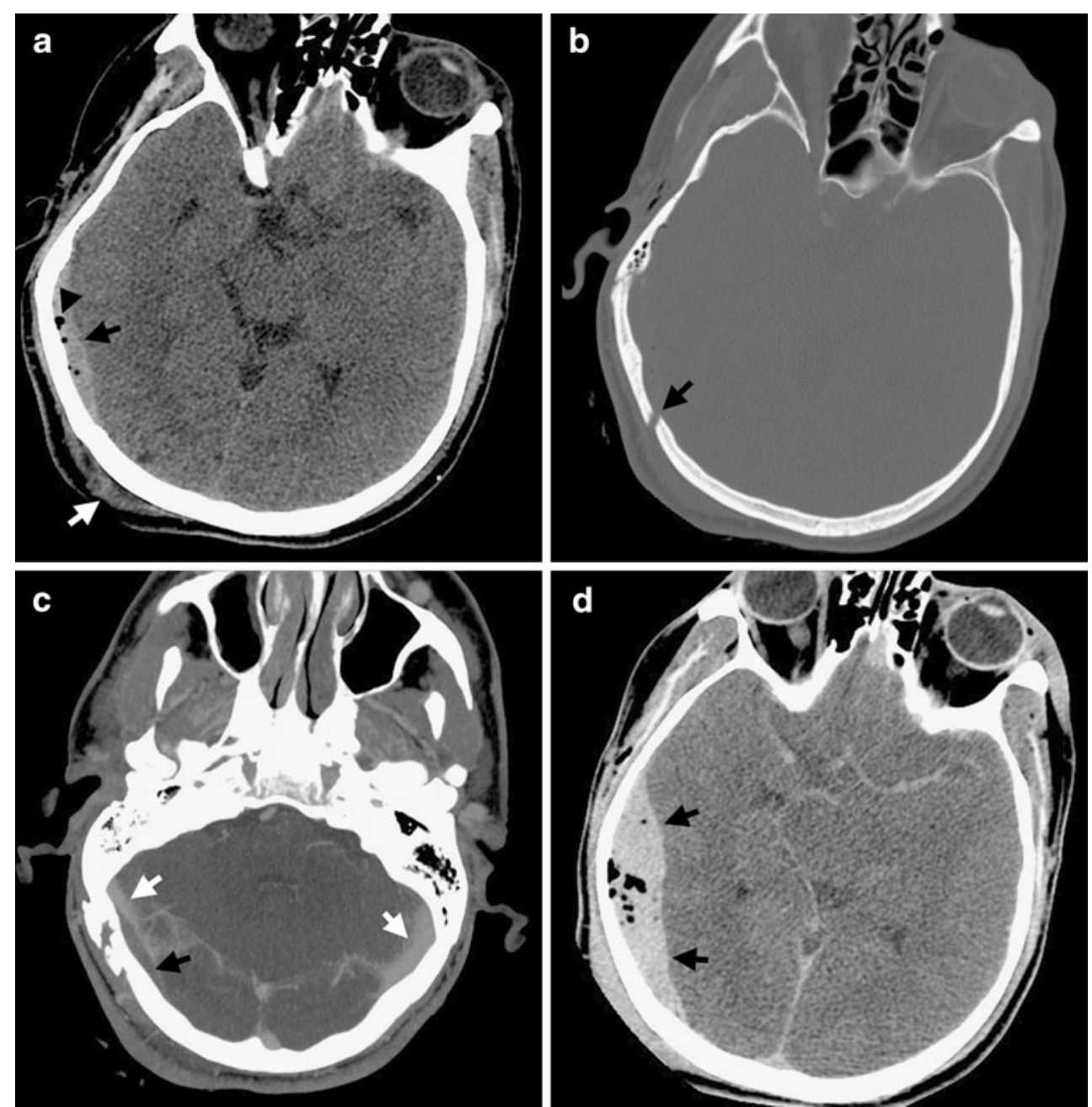

FIG. 2. Venous epidural hematoma. (a) This patient sustained a blow to the back of his right occiput (coup site), as indicated by scalp soft tissue swelling (white arrow). Underlying the coup site is a lentiform EDH (black arrow). Several foci of pneumocephalus are noted (arrowhead) that indicate an associated skull fracture. (b) The displaced skull fracture (black arrow) is best seen on bone windows. (c) Contrast-enhanced CT venogram was obtained as the fracture line extended over the expected location of the right transverse sinus. The opacified transverse sinuses (white arrows) are patent, but the right transverse sinus is compressed and displaced from the inner table of the skull by the EDH (black arrow) caused by injury to the transverse sinus. Note that EDHs form superficial to the periosteal dural layer vesting the outer margin of the venous sinus, thereby possibly displacing the venous sinus away from the calvarium. (d) Follow-up CT $3 \mathrm{~h}$ after presentation shows substantial enlargement of the EDH (black arrows), which is less commonly seen with venous EDHs as compared to arterial EDHs. This was subsequently surgically evacuated. (High resolution version of this image is available in the electronic supplementary material.)
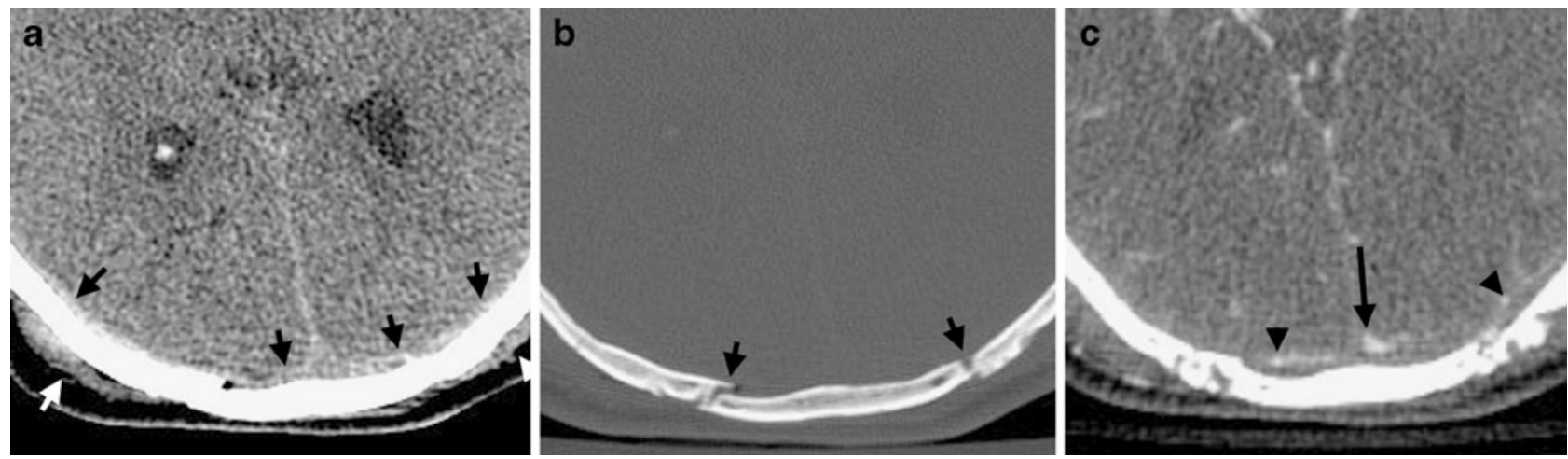

FIG. 3. EDH crossing midline. (a) Underlying the coup site, as indicated by soft tissue swelling (white arrows), is a subtle, relatively thin extra-axial hematoma (black arrows). Note that this hematoma extends across the midline, which distinguishes an EDH from an SDH. (b) The associated calvarial fractures (black arrows) at the coup site are readily appreciated on bone windows. (c) The superior sagittal sinus (long arrow) is displaced from the inner table of the skull by the EDH, as are small cortical vessels (arrowheads). SDH $=\mathrm{Subdural}$ hematoma. (High resolution version of this image is available in the electronic supplementary material.) 
Table 1. Acute Epidural Hematoma, Management Considerations [16]

Surgical evacuation if:

GCS $\leq 8$ with anisocoria, or

Any GCS with EDH volume $>30 \mathrm{~cm}^{3 *}$

Nonoperative monitoring if:

GCS $>8$, and

No focal neurologic deficit, and

$\mathrm{EDH}$ volume $<30 \mathrm{~cm}^{3 *}$, and

EDH thickness $<15 \mathrm{~mm}$, and

Midline shift $<5 \mathrm{~mm}$

GCS $=$ Glasgow Coma Scale; EDH $=$ epidural hematoma

* Hematoma volume can be calculated by the $A B C / 2$ method described by Kothari et al [21].

\section{Subarachnoid hemorrhage}

Subarachnoid hemorrhage (SAH) occurs in $\sim 40 \%$ of patients with moderate to severe head injury and is commonly associated with other types of intracranial hemorrhage [24]. SAH may result from direct laceration of the small cortical vessels traversing the subarachnoid space, redistribution of intraventricular hemorrhage exiting the fourth ventricular outflow foramen, or direct extension from cortical contusion/hematoma. Patients with traumatic SAH have a significantly worse outcome than those without SAH: $41 \%$ of patients without traumatic SAH achieved a level of good recovery compared with only $15 \%$ of patients with SAH, according to the results of a large study conducted by the European Brain Injury Consortium [25].

Acute SAH is readily identified on noncontrast CT scans as linear areas of high attenuation in the cerebral sulci at the convexities, Sylvian fissures, or basilar cisterns. It is important to examine areas such as the interpeduncular cistern, which may contain subtle SAH that is easily missed without conscious attention to this area. In $\sim 5 \%$ of patients with TBI, SAH may be the only abnormal finding on a noncontrast CT scan [25].

Hydrocephalus is a common complication of traumatic SAH and may develop acutely or in a delayed fashion. Intraventricular hemorrhage or inflammatory arachnoiditis may result in hydrocephalus in the acute setting, while decreased resorption of CSF by arachnoid villi is responsible for chronic hydrocephalus.

\section{Cortical contusion/hematoma}

Cortical contusions in closed head injury result when the brain is bruised by the irregular inner surfaces of the skull at the time of impact. They can occur at the coup site when a depressed skull fracture or transient calvarial deformity from a blow to the stationary head grazes the underlying cortex. More commonly, however, they occur at the contrecoup location when the moving head collides against a stationary object. These contrecoup contusions are frequently of greater severity than the injuries at the coup site. The precise mechanism of contrecoup contusions and the explanation for their greater severity are subjects of considerable debate. A recent explanation proposes that when a moving skull collides against a stationary object, such as when a person falls and strikes the back of his head against the ground, the buoyant brain is displaced by CSF in the opposite direction from impact, causing injury opposite the blow [26].

Contrecoup injuries are especially severe when the blow occurs to the occiput, allowing the rough, irregular surfaces of the anterior and central skull base to bruise the undersurface of the brain, resulting in the characteristic hemorrhagic contusions seen in the inferior frontal and temporal lobes (FIG. 6). Contusions appear as small, focal areas of petechial hemorrhage peripherally located
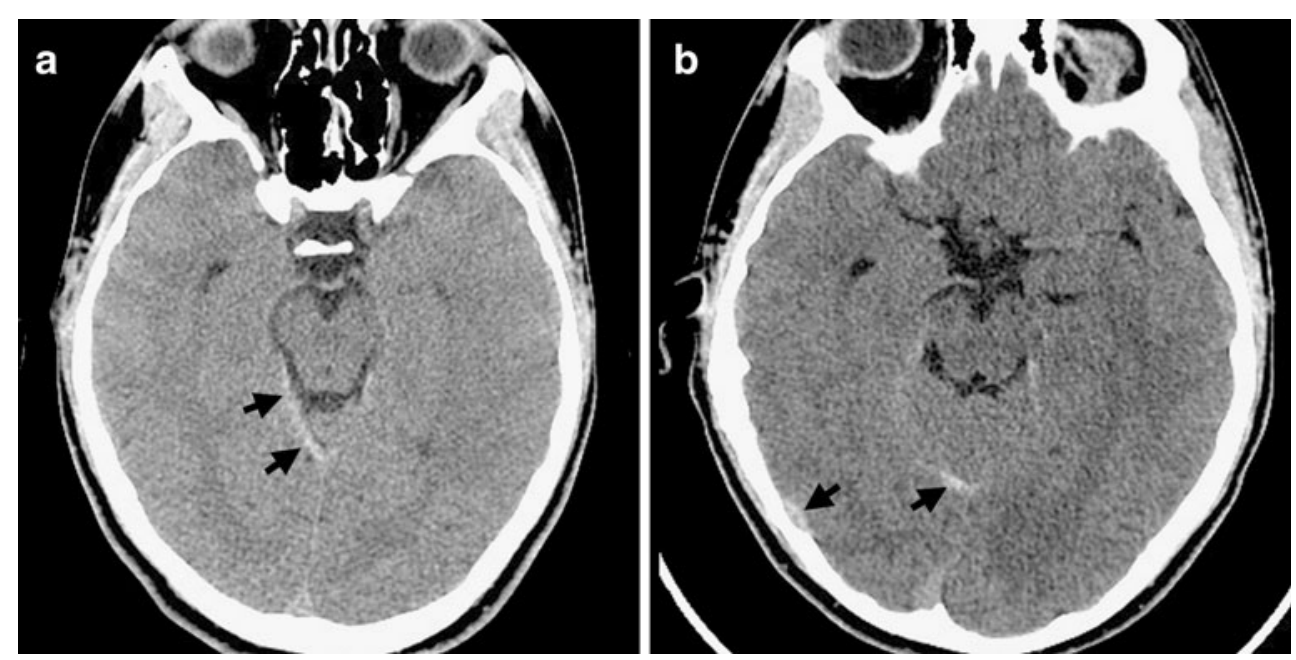

FIG. 4. Subtle tentorial subdural hematoma. (a) There is relatively subtle, asymmetric high density and thickening (arrows) along the right margin of the tentorial incisura as compared to the left, consistent with a very small SDH. This was the only intracranial abnormality in this young patient with minor head trauma. (b) Follow-up CT within $6 \mathrm{~h}$ demonstrates redistribution of the SDH, which can now be seen overlying the cerebral convexity in addition to the tentorium (arrows). (High resolution version of this image is available in the electronic supplementary material.) 

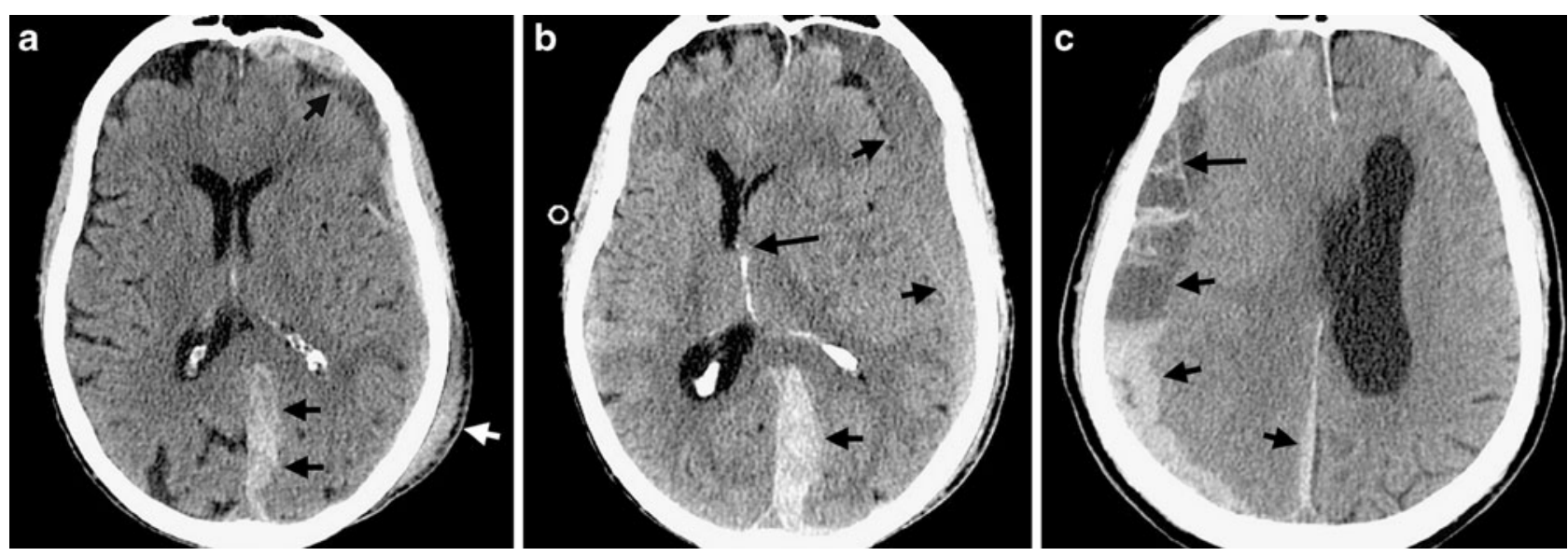

FIG. 5. Heterogeneous subdural hematomas. (a) This patient suffered a blow to the left side of her head (white arrow) and has a left-sided holohemispheric SDH (black arrows) that extends along the falx posteriorly. Note the heterogeneity of attenuation, with mixed high- and low-density areas that are worrisome indications of active bleeding or an underlying coagulopathy. Note also the mass effect of the SDH, which compresses the underlying brain parenchyma and causes asymmetric sulcal effacement. (b) Follow-up CT scan $8 \mathrm{~h}$ later shows substantial enlargement of the SDH (short arrows) as would be expected with active bleeding or coagulopathy, with a new left-to-right midline shift (long arrow). (c) Noncontrast CT scan in a different patient also shows a heterogeneous, holohemispheric SDH (short arrows). In this case, however, heterogeneity is caused by acute hemorrhage into a pre-existing chronic SDH, as indicated by fibrinous strands (long arrow), which are commonly seen with chronic SDHs. (High resolution version of this image is available in the electronic supplementary material.)

in the brain, but they may be very subtle on initial noncontrast $\mathrm{CT}$ scans with minimal hemorrhage and/or edema. However, over time, approximately half of contusions evolve and grow larger in size (FIG. 7) [27]. For this reason, serial CT imaging and close clinical monitoring of TBI patients is paramount. Gradient-echo (GRE) sequences on MRI, which are particularly sensitive to inhomogeneities in the magnetic field caused by paramagnetic hemorrhage, are far more sensitive than $\mathrm{CT}$ in detecting small hemorrhagic contusions.

With greater severity of head trauma, microhemorrhages associated with a contusion may coalesce into an intracerebral hematoma (ICH), reflecting that contusions and ICH represent a spectrum of injury rather than discrete, independent entities. ICHs may also develop in delayed fashion in a part of the brain that was previously seen to be radiographically normal, an entity known as the "delayed traumatic intracerebral hematoma." Similar to contusions, ICHs represent a dynamic process of injury. According to one study, only $56 \%$ of ICH $>3 \mathrm{~cm}$

Table 2. Acute Subdural Hematoma, Management Considerations [23]

Surgical evacuation in all patients regardless of GCS, if:

SDH thickness $>10 \mathrm{~mm}$, or

Midline shift $>5 \mathrm{~mm}$

Nonoperative monitoring in patients with GCS $\leq 8$, if:

SDH thickness $<10 \mathrm{~mm}$, and

Midline shift $<5 \mathrm{~mm}$, and

ICP $<20 \mathrm{~mm} \mathrm{Hg}$, and

Neurologically stable, and

No pupillary abnormalities

$\mathrm{SDH}=$ Subdural hematoma; ICP $=$ intracranial pressure. in diameter developed within $6 \mathrm{~h}$ of injury, and only $84 \%$ of ICH reached maximal size by $12 \mathrm{~h}$, [28] underscoring again the importance of intensive clinical monitoring and serial imaging.

The specific indications for craniotomy and surgical evacuation in patients with contusion or ICH have not been established. Generally, a combination of clinical and radiologic factors is felt to be important, including hemorrhage location and volume, extent of mass effect on CT (cisternal effacement or midline shift), GCS score, ICP, and neurological deterioration [29-32]. Patients with contusions/ICH and progressive neurological decline, medically refractory increased ICP, or radiographic evidence of mass effect tend to have poor outcome without surgical treatment, although specific surgical criteria have not been established.

\section{Traumatic axonal injury}

Traumatic axonal injury (TAI) is also commonly referred to as diffuse axonal injury or shear injury. The term TAI is preferred by the authors of this review as injuries are not distributed diffusely throughout the whole brain, but occur in characteristic, discrete locations, including the parasagittal white matter near the cerebral cortex, corpus callosum, and brainstem. Mild (grade I) TAI involves the gray-white junction of the lobar white matter, particularly the parasagittal frontal lobes; moderate (grade II) TAI involves the fibers of the corpus callosum, particularly the splenium, in addition to the subcortical white matter; severe (grade III) TAI involves the dorsolateral midbrain, in addition to the subcortical white matter and corpus callosum. 


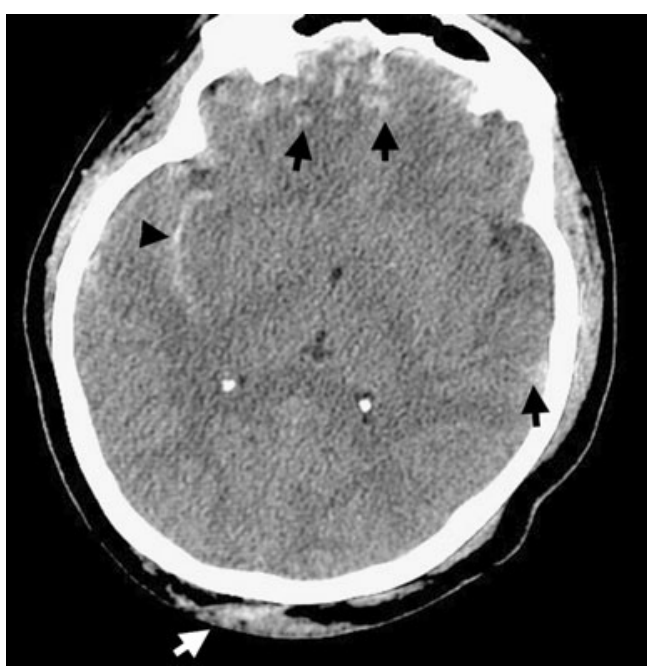

FIG. 6. Hemorrhagic contusions. Following a blow to the occiput, as indicated by soft tissue swelling (white arrow), characteristic contrecoup hemorrhagic contusions are seen in the inferior frontal and temporal lobes (black arrows). Also note the SAH (arrowhead) in the right Sylvian fissure, which is a poor prognostic indicator. SAG = Subarachnoid hemorrhage. (High resolution version of this image is available in the electronic supplementary material.)

The mechanism of injury is one of cytoplasmic shear-strain of the axonal cytoskeleton due to sustained acceleration/deceleration, such as that which occurs with a high-speed motor vehicle crash or prolonged shaking [33]. Damage to the neurons occurs not only at the time of mechanical injury but in the hours, days, and weeks, even years, following the traumatic event due to a deleterious cascade of biochemical events and Wallerian-type degeneration with progressive neuronal loss. Changes associated with TAI are thought to be responsible for the majority of global cognitive defects seen after TBI, particularly with regard to difficulties with memory and information processing.

Unfortunately, TAI is notoriously difficult to diagnose by CT. Only $10 \%$ of TAI patients demonstrate the classic CT findings of petechial hemorrhages at the gray-white junction of the cerebral hemispheres, corpus callosum, or brainstem. Indeed, the results of most admission CT exams are normal because $>80 \%$ of TAIs are nonhemorrhagic [33, 34]. MRI is far more sensitive than CT as it can detect both hemorrhagic and nonhemorrhagic lesions (FIG. 8). Acute hemorrhagic lesions demonstrate focal susceptibility and signal loss due to the paramagnetic effects of deoxyhemoglobin on gradientrecalled echo (GRE) and susceptibility-weighted images (SWI). These susceptibility effects are further magnified at 3 Tesla (3T) compared to $1.5 \mathrm{~T}$, with nearly twice as many hemorrhagic lesions detectable at 3T [35]. Nonhemorrhagic lesions are best appreciated on fluid attenuation inversion recovery imaging, which makes subcortical and periventricular lesions particularly conspicuous (as compared with conventional T2-weighted imaging). Diffusion-weighted imaging (DWI) is also sensitive for TAI and can detect lesions that are not seen on either fluid attenuation inversion recovery or GRE sequences (FIG. 9) [36]. TAI lesions typically appear hyperintense with reduced apparent diffusion coefficient values in the acute setting, likely reflecting cytotoxic edema [37]. Unfortunately, there are no good treatment options for TAI patients at this time, and the value of improving the accuracy of imaging diagnosis lies chiefly in the ability to better prognosticate clinical outcome, as will be discussed in further detail later in this review.
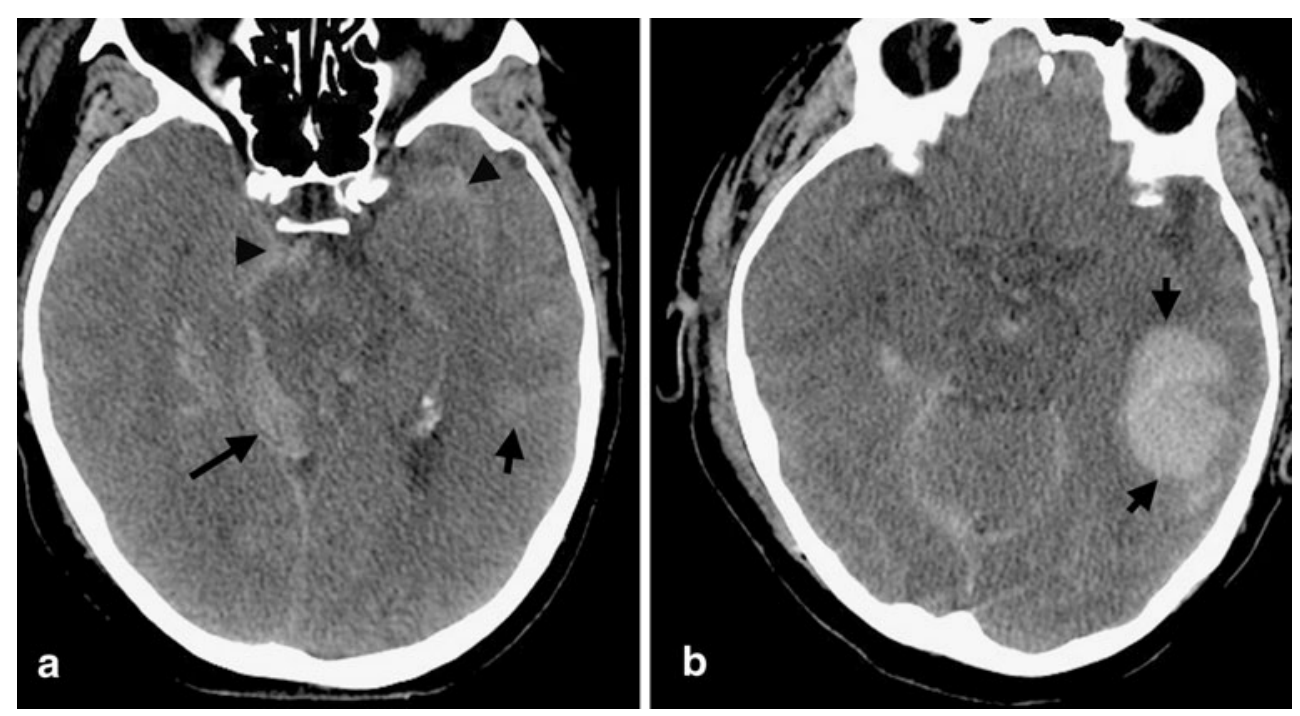

FIG. 7. Blossoming of hemorrhagic contusions. (a) Multiple intracranial hemorrhages are seen in this patient, including a small, subtle left temporal hemorrhagic contusion (short arrow), SDH along the right tentorium (long arrow), and SAH in the basilar cisterns and Sylvian fissure (arrowheads). (b) Follow-up CT scan $6 \mathrm{~h}$ later demonstrates significant expansion of the left temporal contusion (short arrows) into an intraparenchymal hematoma, underscoring the importance of serial CT monitoring. (High resolution version of this image is available in the electronic supplementary material.) 

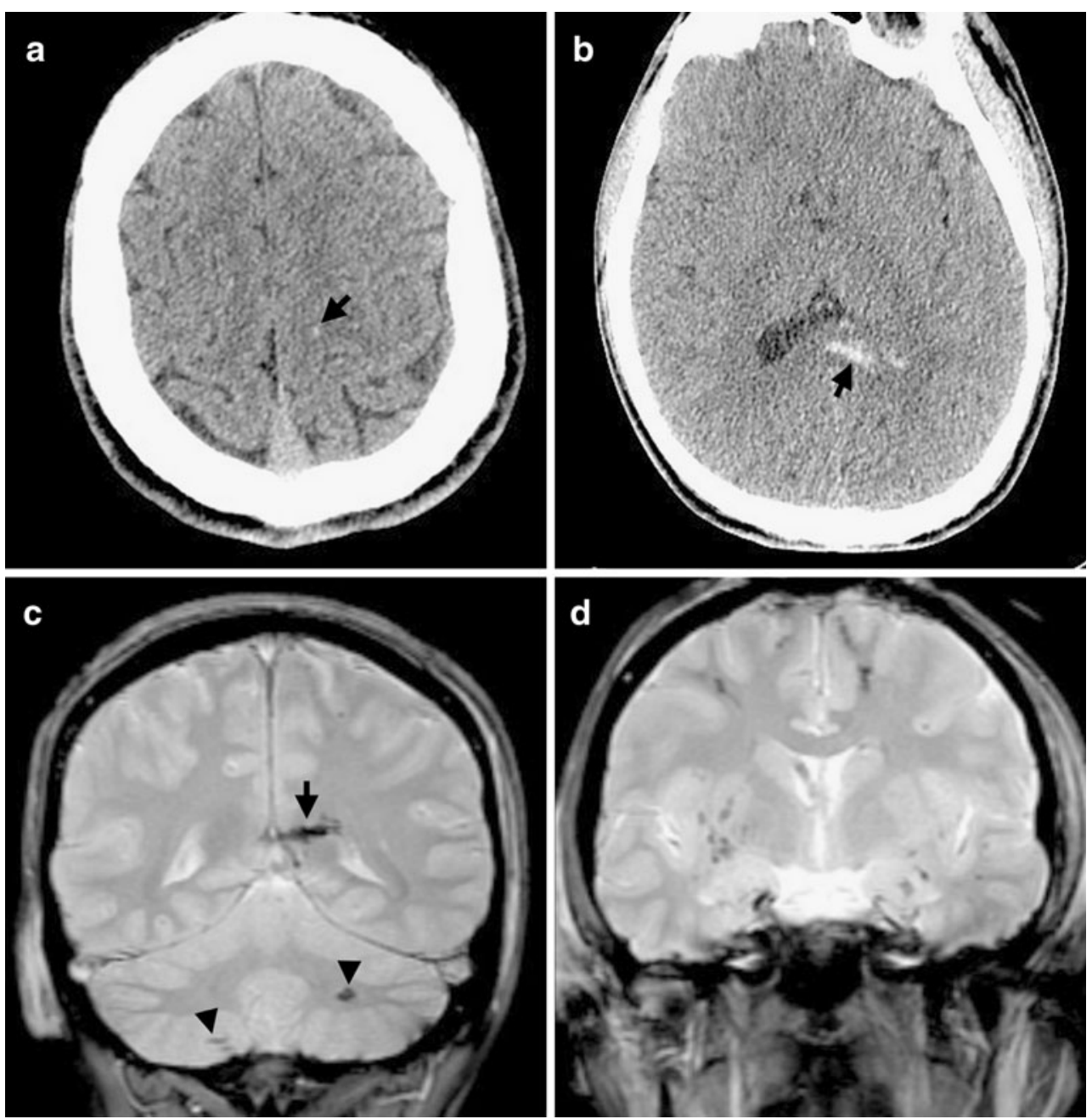

FIG. 8. Traumatic axonal injury on CT and MRI. Small foci of hemorrhagic TAl are seen in the right parasagittal posterior frontal lobe (arrow, a) and in the splenium of the corpus callosum (arrow, b). No other foci of TAl were observed on this admission CT scan. However, coronal gradient-echo images from a subsequent MRI scan obtained three days later at 1.5T demonstrate susceptibility from hemorrhagic TAl not only in the splenium (arrow, c) but also in the cerebellar hemispheres (arrowheads, c). Many other foci of TAl are seen in the basal ganglia and at the gray-white junction of both the frontal and temporal lobes (d) illustrating the superior sensitivity of MRI over to CT for TAI. $\mathrm{MRI}=$ Magnetic resonance imaging. (High resolution version of this image is available in the electronic supplementary material.)

\section{SECONDARY INJURIES IN TBI PATIENTS}

\section{Cerebral swelling}

There is an ongoing debate in the literature as to whether cerebral edema or cerebral hyperemia with increased blood volume is the underlying cause of cerebral swelling. Both mechanisms may be at play. A generally held premise is that cerebral hyperemia due to dysautoregulation with vascular engorgement and increased cerebral blood volume is the principal mechanism of cerebral swelling, although recent data suggest that blood volume may actually be decreased following trauma and that cerebral edema may be the major fluid component of brain swelling [38, 39]. There are two types of cerebral edema: vasogenic, due to disruption of the blood-brain barrier allowing accumulation of extracellular water, and cytotoxic, due to the failure of cell membrane pumps, resulting in intracellular water leakage. Of these two types of edema, cytotoxic edema may be primarily responsible for cerebral swelling, as demonstrated by the increased water content and reduced apparent diffusion coefficient values in one MRI study [40].

On imaging, cerebral swelling due to hyperemia is represented as the loss of sulci (sulcal effacement), compression of the basilar cisterns, and flattening of the ventricular margins (FIG. 10). Gray and white matter attenuation and differentiation will remain intact. However, cerebral swelling due to vasogenic edema will be revealed as areas of low attenuation, while swelling due to cytotoxic edema will appear as a loss of gray-white differentiation.

Children are particularly susceptible to diffuse cerebral swelling following TBI, with the incidence of diffuse 

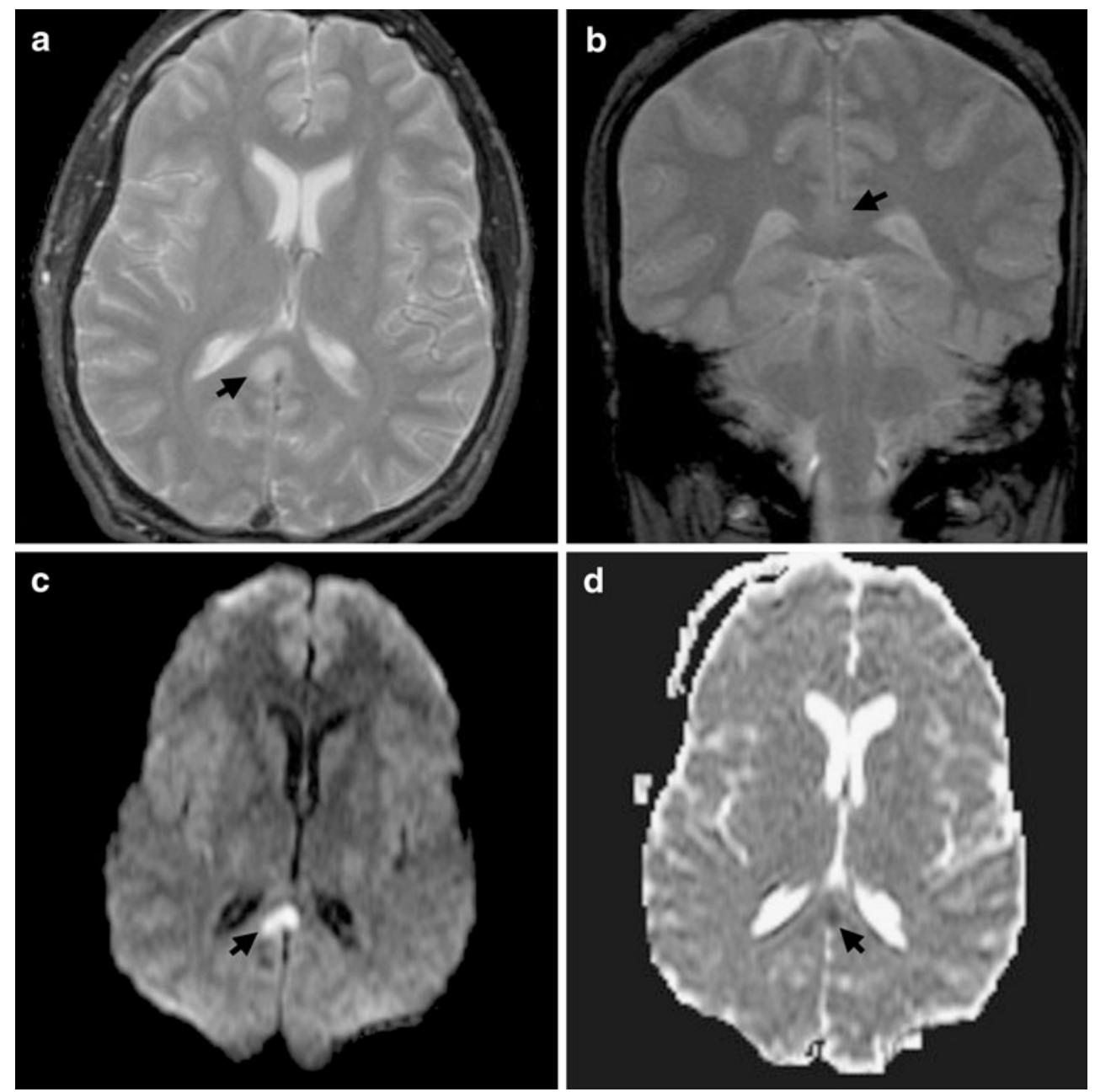

FIG. 9. Traumatic axonal injury on diffusion-weighted imaging. (a) Axial T2-weighted spin echo sequence demonstrates hyperintensity in the splenium of the corpus callosum (arrow), a characteristic location for TAl. (b) Coronal gradient-recall echo sequence shows hyperintensity in the splenium (arrow) but no susceptibility artifact to suggest hemorrhage. (c) and (d) The signal abnormality (arrow) is much more conspicuous on diffusion-weighted imaging (c) and has reduced diffusion on the apparent diffusion coefficient map (d) consistent with cytotoxic edema in this focus of non-hemorrhagic TAl. (High resolution version of this image is available in the electronic supplementary material.)

swelling being approximately twofold higher in children than in adults [41]. Children and young adults are more prone to post-traumatic dysautoregulation, which leads to vasodilatation, hyperemia, and cerebral swelling. When swelling is severe, ICP increases and CPP falls, resulting in infarction and cerebral damage.

Medical strategies to combat the deleterious effects of cerebral swelling and lower ICP include head elevation, moderate hyperventilation, hyperosmolar therapy with mannitol, and the appropriate use of sedatives and analgesics (barbiturates) to prevent pain or agitation from exacerbating elevated ICP. However, when ICP is refractory to maximal medical therapy, decompressive craniectomy has been used in an attempt to lower ICP. Studies on the efficacy of decompressive craniectomy generally suggest that it can lower ICP and may improve functional outcome [42-44]. Decompressive craniectomy also results in radiologic improvement, with better visualization of basilar cisterns and decreased midline shift, both of which correlate with improved outcome [45]. However, the specific clinical criteria and context for decompressive surgery have yet to be established.

\section{Cerebral herniation}

Cerebral herniation is a result of unmitigated increased ICP. These patients typically undergo decompressive craniectomy with mass lesion evacuation.

Subfalcine herniation or midline shift occurs when the cingulate gyrus herniates under the falx cerebri; uncal herniation results when the medial temporal lobe herniates through the tentorial incisura and compresses the ipsilateral suprasellar cistern; descending transtentorial herniation occurs with downward herniation of both temporal lobes through the tentorial incisura, compressing the basilar cisterns; upward transtentorial herniation occurs in the opposite direction, with the cerebellum 

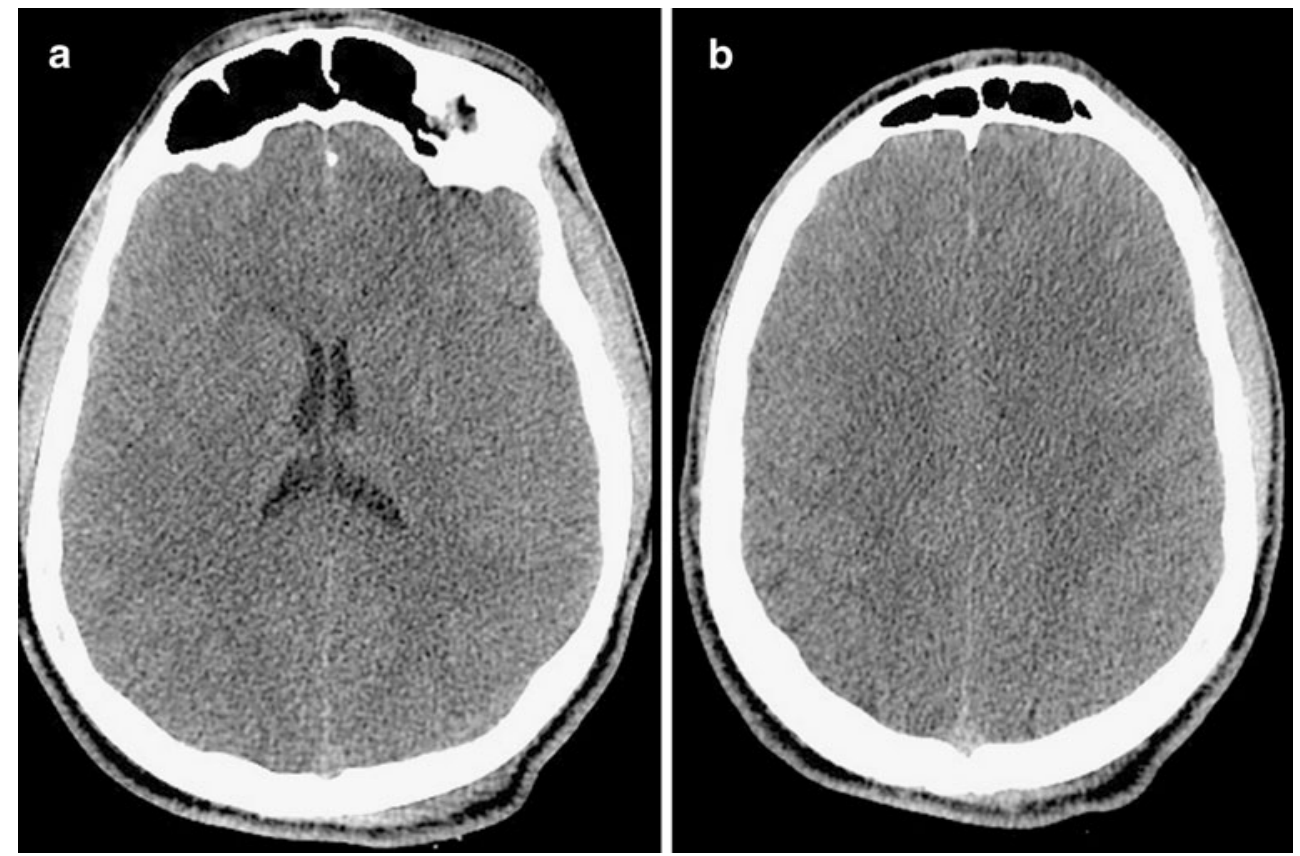

FIG. 10. Diffuse cerebral swelling. (a) and (b) Axial noncontrast CT scans demonstrate diffuse sulcal effacement in this 32-year-old patient who sustained head trauma. Note the absence of cerebral sulci but the relative preservation of gray-white differentiation, suggesting cerebral hyperemia due to post-traumatic dysautoregulation. (High resolution version of this image is available in the electronic supplementary material.)

extending through the tentorial incisura and effacing the quadrigeminal cistern; tonsillar herniation results when the cerebellar tonsils herniate into the foramen magnum.

In the absence of CT imaging, any patient with a blown pupil is clinically presumed to have cerebral herniation. With uncal herniation, the displaced medial temporal lobe compresses the ipsilateral oculomotor nerve, resulting in loss of the normal pupillary reflex. Other important sequelae of cerebral herniation include cerebral infarction, which will be discussed in the following section, and the Duret hemorrhage, which typically occurs in the ventral and paramedian midbrain/ pons following rapid downward herniation (FIG. 11). It is thought that either laceration of the pontine perforator branches from the basilar artery or venous thrombosis with infarct is the underlying mechanism of injury in the Duret hemorrhage.

\section{Cerebral ischemia and infarction}

Cerebral ischemia and infarction occurs in $2 \%$ of patients undergoing head CT for TBI and can occur from a variety of causes [46]. Most commonly, ischemia occurs due to mechanical compression of a blood vessel from cerebral herniation across the falx cerebri and/or tentorium. Infarctions due to mechanical shift are typically seen in the anterior cerebral artery or posterior cerebral artery distribution following acute subfalcine or uncal herniation, respectively (FIG. 12). The callosomarginal branch of the anterior cerebral artery can be compressed against the free edge of the falx and cause infarction with subfalcine herniation. In uncal herniation, the displaced medial temporal lobe can compress the PCA, resulting in infarction, or constrict the anterior choroidal artery, leading to infarction of the posterior limb of the internal capsule.

Other potential causes of ischemia and infarction in patients with TBI include vasospasm, which has been angiographically documented in patients following head trauma.47 Also, extra-axial hematomas that exert a significant mass effect on the adjacent cortex may compress cortical veins and result in venous infarction. Finally, direct vascular injury, such as dissection, occlusion, or pseudoaneurysm from a skull base fracture, may also result in ischemia.

\section{ROLE OF IMAGING \\ IN THE PROGNOSTICATION OF OUTCOME FOLLOWING TBI}

In addition to diagnosing and guiding the management of TBI, imaging modalities can be used to predict clinical and functional outcome after head injury. Because noncontrast CT has been the mainstay of imaging acute TBI given its speed, accessibility, and sensitivity to hemorrhage, attention has historically focused on identifying CT predictors of clinical outcome. The widely used Marshall classification and Rotterdam score (discussed below) relate imaging findings on noncontrast CT scans to mortality and patient outcome. However, with the development of novel $\mathrm{CT}$ techniques, such as $\mathrm{CT}$ 

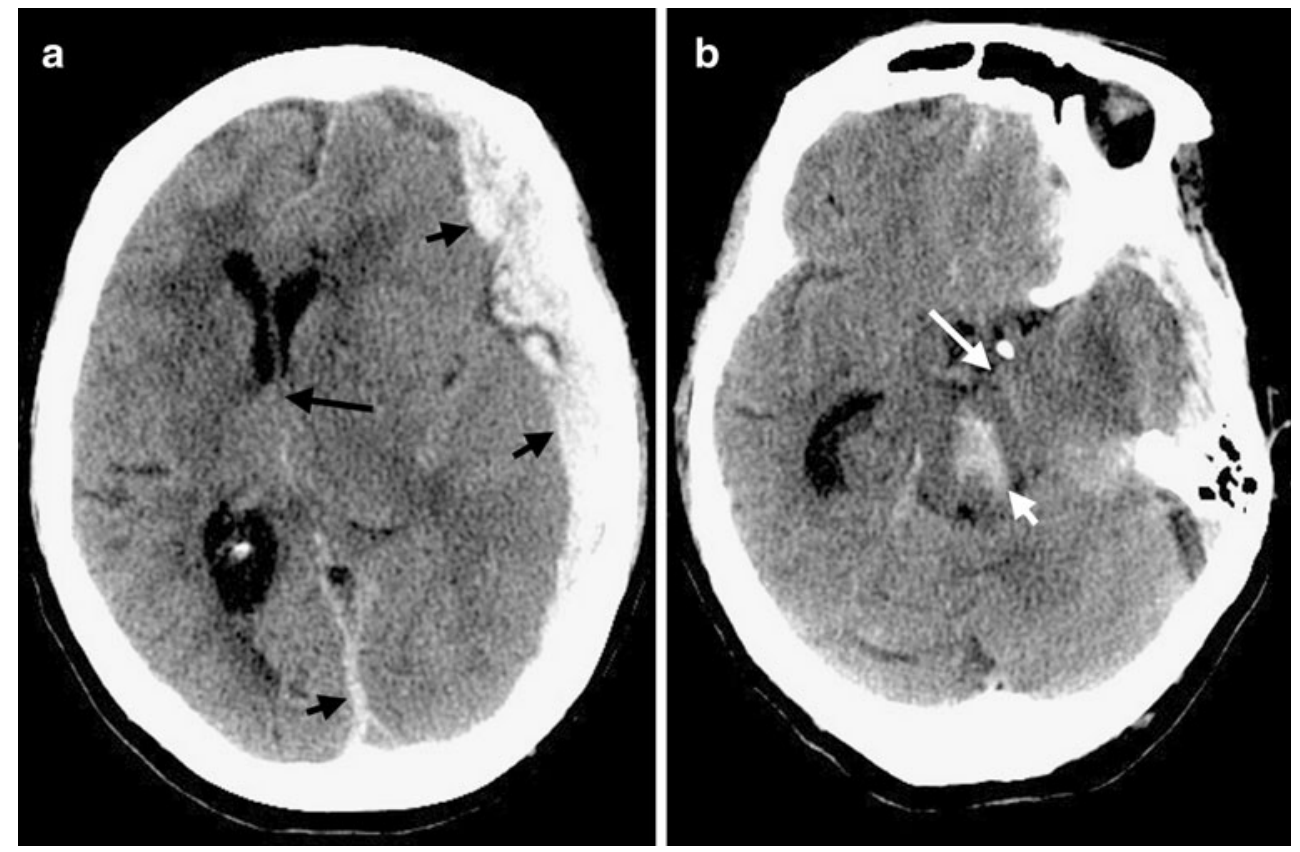

FIG. 11. Duret hemorrhage with cerebral herniation. Large left holohemispheric and parafalcine subdural hematoma (short black arrows, a) results in midline shift (long black arrow, a) and uncal (long white arrow, b) herniation. Downward brainstem herniation has led to classic Duret hemorrhage (short white arrow, b) in the paramedian midbrain. (High resolution version of this image is available in the electronic supplementary material.)

perfusion, there is considerable interest in investigating these modalities to better treat and prognosticate TBI patients. As with CT, continued innovations in MRI technology have led to developments such as susceptibility-weighted and diffusion tensor imaging that may also help to better prognosticate outcome in TBI patients.

\section{Routine noncontrast CT}

Individual predictors of poor outcome on conventional CT include compressed or absent basilar cisterns, the presence of subarachnoid hemorrhage, midline shift, and intracranial hemorrhagic lesions (e.g., subdural or epidural hematomas, shear injury, and contusions). Compressed or absent basilar cisterns indicate a threefold higher risk of increased ICP and are associated with a two- to threefold increase in mortality [48]. Traumatic subarachnoid hemorrhage is associated with a twofold increase in mortality, and hemorrhage in the basilar cisterns has a $70 \%$ positive predictive value for poor outcome [48]. Midline shift indicates increased ICP and is similarly associated with a poor clinical outcome, although this association is somewhat complicated by the fact that midline shift is caused by intracranial hemorrhage that also negatively impacts outcome. Finally, intracranial hemorrhage has an approximately $80 \%$ positive predictive value for poor functional outcome, with prognosis worsening as the hematoma volume increases in size [48]. Of note, mortality is higher in patients with acute subdural hematomas compared with those with epidural hematomas.
Marshall et al., [49] in his publication on the Trauma Coma Data Bank, aggregated key findings on CT and proposed an imaging-based classification system of head injury that predicts mortality (Table 3). This system defines 4 types of diffuse head injury and takes the presence of a mass lesion and evidence of increased ICP (status of the basilar cisterns, presence of midline shift) into account. The Marshall Classification is widely recognized and used, but a more recent study published by Maas et al. [50]. from Rotterdam further refined imaging parameters to develop a CT score that better predicts clinical outcome. The Rotterdam Score evaluates the status of the basilar cisterns, presence of midline shift, presence of epidural hematoma, and presence of intraventricular or subarachnoid hemorrhage to derive a score that relates to 6-month mortality (Table 4).

\section{Advanced CT techniques}

While noncontrast CT is invaluable for the diagnosis, management and prognosis of patients with TBI, it is limited to displaying only anatomic information. Physiologic derangements in cerebral perfusion, blood flow, and oxygenation related to the deleterious cascade of secondary traumatic brain injuries that significantly impacts functional outcome are not captured on conventional noncontrast CT scans. Perfusion CT is a new imaging tool that utilizes dynamic scanning during intravenous contrast injection to illustrate physiologic parameters of cerebral blood volume (CBV), cerebral blood flow 


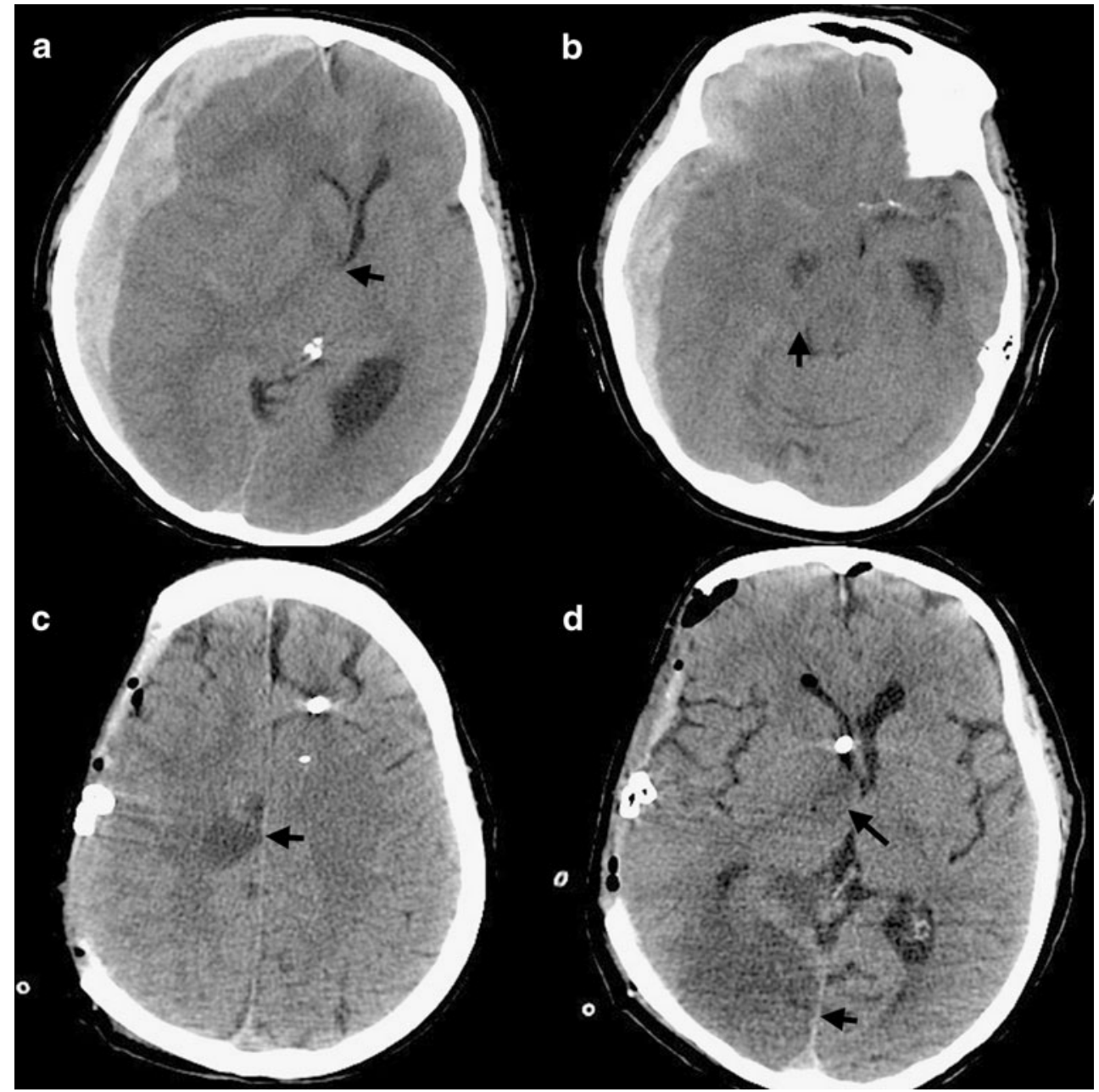

FIG. 12. Infarction as complication of cerebral herniation. Large right-sided holohemispheric subdural hematoma results in subfalcine (arrow, a) and uncal (arrow, b) herniation. Despite decompressive hemicraniectomy, the patient subsequently developed infarcts in the anterior cerebral artery (arrow, c) and posterior cerebral artery (short arrow, d) distributions due to subfalcine and uncal herniation, respectively. Note also infarction of the posterior limb of the right internal capsule (long arrow, d) which is due to compression of the anterior choroidal artery with uncal herniation. (High resolution version of this image is available in the electronic supplementary material.)

(CBF), and mean transit time (time for blood to perfuse a region of tissue).

Evidence of normal brain perfusion or hyperemia (high $\mathrm{CBV}$ and $\mathrm{CBF}$ ) on perfusion CT scans in TBI patients has been associated with a favorable clinical outcome, while oligemia (low $\mathrm{CBV}$ and $\mathrm{CBF}$ ) has been associated with unfavorable outcome [51]. These findings on perfusion $\mathrm{CT}$ scans have been postulated to reflect the status of cerebrovascular autoregulation: patients with relatively minor head trauma and intact autoregulation maintain or even slightly increase cerebral perfusion, while patients with severe head injury have impaired

Table 3. Marshall Classification of Head Injury (Trauma Coma Data Bank)

\begin{tabular}{|c|c|c|}
\hline Category & Definition & Mortality ${ }^{*}$ \\
\hline Diffuse injury I (no visible pathology) & No visible intracranial pathology on CT scan & $10 \%$ \\
\hline Diffuse injury II & $\begin{array}{l}\text { Cisterns present with midline shift } \leq 5 \mathrm{~mm} \text { and/or lesion } \\
\text { densities present, no high- or mixed-density lesion }>25 \mathrm{cc} \text {, } \\
\text { may include bone fragments and foreign bodies }\end{array}$ & $14 \%$ \\
\hline Diffuse injury III & $\begin{array}{l}\text { Cisterns compressed or absent with midline shift } \leq 5 \mathrm{~mm} \text {, } \\
\text { no high- or mixed-density lesion }>25 \mathrm{cc}\end{array}$ & $34 \%$ \\
\hline Diffuse injury IV & Midline shift $>5 \mathrm{~mm}$, no high- or mixed-density lesion $>25 \mathrm{cc}$ & $56 \%$ \\
\hline Evacuated mass lesion & Any lesion surgically evacuated & $39 \%$ \\
\hline Nonevacuated mass lesion & High- or mixed-density lesion $>25 \mathrm{cc}$, not surgically evacuated & $53 \%$ \\
\hline
\end{tabular}

\footnotetext{
${ }^{*}$ Mortality at discharge.
} 
Table 4. Rotterdam Score for Probability of Mortality in Patients with Traumatic Brain Injury

\begin{tabular}{lc}
\hline \multicolumn{1}{c}{ Predictor } & Score \\
\hline Basal cisterns & 0 \\
Normal & 1 \\
Compressed & 2 \\
Absent & 0 \\
Midline shift & 1 \\
No shift or shift $\leq 5 \mathrm{~mm}$ & \\
Shift $>5$ mm & 0 \\
Epidural mass lesion & 1 \\
Present & \\
Absent & 0 \\
Intraventricular or subarachnoid hemorrhage & 1 \\
Absent & \\
Present & +1 \\
Sum score & \\
* The sum score predicts 6-month mortality as follows: & $1=0 \%$ \\
mortality; 2=7\% mortality; $3=16 \%$ mortality; $4=26 \%$ mortality; \\
5=53\% mortality; $6=61 \%$ mortality.
\end{tabular}

autoregulation and pressure-passive flow, which often results in oligemia and decreased perfusion. The excitement of using perfusion $\mathrm{CT}$ to better understand the physiology of TBI patients is tempered by concerns of increased radiation exposure, particularly in young patients who are often the victims of head injury.

\section{Advanced MRI techniques}

While CT is useful for the diagnosis and prognostication of outcome in patients with hemorrhagic injuries such as subdural and epidural hematomas, patients with subarachnoid hemorrhage and contusions, a subset of TBI, have normal CT scans without hemorrhage yet still fare poorly. TAI patients can suffer significant post-traumatic symptoms and may be profoundly comatose, with cognitive impairment and poor functional outcome despite normal results on conventional CT or MR images. As such, there is considerable interest in developing more sensitive diagnostic tests for TAI and for correlating these tests with long-term functional outcome. Unfortunately, as there are no good treatment options for TAI patients at this time, the value of improved imaging diagnosis currently lies in the potential to better prognosticate clinical outcome.

Diffusion tensor imaging (DTI) is one investigative MRI technique that maps out the microstructural characteristics of the brain based on the intrinsic diffusion properties of neurons. Unlike conventional diffusion weighted imaging, which encodes diffusion in 3 basic directions, DTI assesses diffusion in at least 6 but typically 25-30 directions, yielding a more complete set of diffusivity information that can be used to deduce axonal orientation and create maps of white matter tracts in the brain. TAI interrupts the cytoskeletal network and impairs axoplasmic transport, causing changes in tissue diffusivity that can be visualized and quantified on DTI [52]. DTI has been shown to be more sensitive than conventional 3T MRI in detecting TAI, and results from recent studies suggest that the extent of damage to white matter structures on DTI may correlate with the extent of cognitive impairment and functional outcome following TBI [53, 54].

SWI is another relatively new MRI technique that increases sensitivity for the detection of TAI. SWI utilizes a high-resolution, velocity-compensated, 3dimensional GRE sequence based on both magnitude and phase data $[55,56]$. It is exquisitely sensitive to susceptibility effects due to microhemorrhages, small veins, and changes in iron content, and more sensitive than conventional GRE sequences for the detection of hemorrhagic TAI, revealing four- to sixfold more microhemorrhages than conventional GRE imaging [57, 58]. Initial studies also suggest that lesion burden on SWI correlates with clinical outcome, including the duration of coma and long-term functional disability [59, 60].

Magnetoencephalography is a functional imaging technique that measures the magnetic field generated by neuronal activation. Unlike normal brain tissue that generates $\alpha$-waves with a frequency of $8-13 \mathrm{~Hz}$, injured neurons produce $\delta$-waves with a low frequency of 1 $4 \mathrm{~Hz}$. This magnetic signal can be measured and localized with high spatial $(2-3 \mathrm{~mm})$ and temporal $(<1 \mathrm{~ms})$ resolution. In TBI patients, magnetoencephalography "slow waves" can be seen in cortical gray matter that is functionally and structurally connected to white matter fiber tracts with axonal shear injury, as demonstrated on DTI, and may be more sensitive than DTI in diagnosing mild TBI [61].

Functional MRI (fMRI) is another functional imaging technique that relies on the relationship between physiologic function, energy consumption, and blood flow to depict brain activity. Most brain fMRI studies rely on a blood oxygen level-dependent signal to demonstrate cerebral function. It is thought that neuronal activity causes an increase in cerebral flood flow and local blood oxygenation with a relative decrease in the amount of deoxyhemoglobin, a product of oxygen consumption. Deoxyhemoglobin is paramagnetic, generating field inhomogeneities that result in signal loss on GRE sequences sensitive to susceptibility effects. Thus, as the relative concentration of deoxyhemoglobin decreases within a region of neuronal activity, an increased signal within this area is seen on GRE images. Blood oxygen leveldependent fMRI studies performed in patients with mild TBI and structurally normal imaging studies have shown differences in brain activation during various tasks as compared with healthy control subjects $[62,63]$. 


\section{SUMMARY}

To understand the role of imaging in TBI, it is important to appreciate that TBI encompasses a heterogeneous group of intracranial injuries and includes both insults at the time of impact and a deleterious secondary cascade of insults that require optimal medical and surgical management. Initial imaging identifies the acute primary insult that is essential to diagnosing TBI, but serial imaging surveillance is also critical to identifying secondary injuries, such as cerebral herniation and swelling, that guide neurocritical management. CT is the mainstay of TBI imaging in the acute setting, but MRI has better diagnostic sensitivity for nonhemorrhagic contusions and shear-strain injuries. Both CT and MRI can be used to prognosticate clinical outcome, and there is particular interest in advanced applications of both techniques that may greatly improve the sensitivity of conventional CT and MRI for both the diagnosis and prognosis of TBI.

Acknowledgments: The authors have no financial interests in the discussed subject matter to disclose.

Open Access This article is distributed under the terms of the Creative Commons Attribution Noncommercial License which permits any noncommercial use, distribution, and reproduction in any medium, provided the original author(s) and source are credited.

\section{REFERENCES}

1. Centers for Disease Control and Prevention. Traumatic brain injury. Available at: http://wwwcdcgov/traumaticbraininjury/statisticshtml Last modified March 17, 2010.

2. Bratton SL, Chestnut RM, Ghajar J, et al. Guidelines for the management of severe traumatic brain injury. IX. Cerebral perfusion thresholds. J Neurotrauma 2007;24 (suppl 1):S59-64.

3. Nordstrom CH, Reinstrup P, Xu W, Gardenfors A, Ungerstedt U. Assessment of the lower limit for cerebral perfusion pressure in severe head injuries by bedside monitoring of regional energy metabolism. Anesthesiology 2003;98:809-814.

4. Chesnut RM, Marshall LF, Klauber MR, et al. The role of secondary brain injury in determining outcome from severe head injury. $\mathbf{J}$ Trauma 1993;34:216-222.

5. Manley G, Knudson MM, Morabito D, Damron S, Erickson V, Pitts L. Hypotension, hypoxia, and head injury: frequency, duration, and consequences. Arch Surg 2001;136:1118-1123.

6. Bratton SL, Chestnut RM, Ghajar J, et al. Guidelines for the management of severe traumatic brain injury. I. Blood pressure and oxygenation. J Neurotrauma 2007;24 (suppl 1):S7-13.

7. Bratton SL, Chestnut RM, Ghajar J, et al. Guidelines for the management of severe traumatic brain injury. VI. Indications for intracranial pressure monitoring. J Neurotrauma 2007;24 (suppl 1):S37-44.

8. Narayan RK, Greenberg RP, Miller JD, et al. Improved confidence of outcome prediction in severe head injury. A comparative analysis of the clinical examination, multimodality evoked potentials, CT scanning, and intracranial pressure. J Neurosurg 1981;54:751-762.

9. Bratton SL, Chestnut RM, Ghajar J, et al. Guidelines for the management of severe traumatic brain injury. VIII. Intracranial pressure thresholds. J Neurotrauma 2007;24 (suppl 1):S55-58.
10. Eisenberg HM, Frankowski RF, Contant CF, Marshall LF, Walker MD. High-dose barbiturate control of elevated intracranial pressure in patients with severe head injury. J Neurosurg 1988;69:15-23.

11. Narayan RK, Kishore PR, Becker DP, et al. Intracranial pressure: to monitor or not to monitor? A review of our experience with severe head injury. J Neurosurg 1982;56:650-659.

12. Schouten JW. Neuroprotection in traumatic brain injury: a complex struggle against the biology of nature. Curr Opin Crit Care 2007; 13:134-142.

13. Maas AI, Roozenbeek B, Manley GT. Clinical trials in traumatic brain injury: past experience and current developments. Neurotherapeutics 2010;7:115-126.

14. Doppenberg EM, Choi SC, Bullock R. Clinical trials in traumatic brain injury: lessons for the future. J Neurosurg Anesthesiol 2004;16:87-94.

15. Zimmerman RA, Bilaniuk LT. Computed tomographic staging of traumatic epidural bleeding. Radiology 1982;144:809-812.

16. Bullock MR, Chesnut R, Ghajar J, et al. Surgical management of acute epidural hematomas. Neurosurgery 2006;58:S7-15; discussion $\mathrm{Si}-\mathrm{iv}$.

17. Gean AD, Fischbein NJ, Purcell DD, Aiken AH, Manley GT, Stiver SI. Benign anterior temporal epidural hematoma: indolent lesion with a characteristic CT imaging appearance after blunt head trauma. Radiology 2010;257:212-218.

18. Lee EJ, Hung YC, Wang LC, Chung KC, Chen HH. Factors influencing the functional outcome of patients with acute epidural hematomas: analysis of 200 patients undergoing surgery. J Trauma 1998;45:946-952.

19. Rivas JJ, Lobato RD, Sarabia R, Cordobes F, Cabrera A, Gomez P. Extradural hematoma: analysis of factors influencing the courses of 161 patients. Neurosurgery 1988;23:44-51.

20. Servadei F, Faccani G, Roccella P, et al. Asymptomatic extradural haematomas. Results of a multicenter study of 158 cases in minor head injury. Acta Neurochir (Wien) 1989;96:39-45.

21. Kothari RU, Brott T, Broderick JP, et al. The ABCs of measuring intracerebral hemorrhage volumes. Stroke 1996;27:1304-1305.

22. Aiken AH, Gean AD. Imaging of head trauma. Semin Roentgenol 2010;45:63-79.

23. Bullock MR, Chesnut R, Ghajar J, et al. Surgical management of acute subdural hematomas. Neurosurgery 2006;58:S16-24; discussion $\mathrm{Si}-\mathrm{iv}$.

24. Murray GD, Teasdale GM, Braakman R, et al. The European Brain Injury Consortium survey of head injuries. Acta Neurochir (Wien) 1999;141:223-236.

25. Servadei F, Murray GD, Teasdale GM, et al. Traumatic subarachnoid hemorrhage: demographic and clinical study of 750 patients from the European brain injury consortium survey of head injuries. Neurosurgery 2002;50:261-267; discussion 267-269.

26. Drew LB, Drew WE. The contrecoup-coup phenomenon: a new understanding of the mechanism of closed head injury. Neurocrit Care 2004;1:385-390.

27. Beaumont A, Gennarelli T. CT prediction of contusion evolution after closed head injury: the role of pericontusional edema. Acta Neurochir Suppl 2006;96:30-32.

28. Yamaki T, Hirakawa $K$, Ueguchi $T$, Tenjin $H$, Kuboyama $T$, Nakagawa Y. Chronological evaluation of acute traumatic intracerebral haematoma. Acta Neurochir (Wien) 1990;103:112-115.

29. Bullock R, Golek J, Blake G. Traumatic intracerebral hematomawhich patients should undergo surgical evacuation? CT scan features and ICP monitoring as a basis for decision making. Surg Neurol 1989;32:181-187.

30. Choksey M, Crockard HA, Sandilands M. Acute traumatic intracerebral haematomas: determinants of outcome in a retrospective series of 202 cases. Br J Neurosurg 1993;7:611-622

31. Servadei F, Murray GD, Penny K, et al. The value of the "worst" computed tomographic scan in clinical studies of moderate and severe head injury. European Brain Injury Consortium. Neurosurgery 2000;46:70-75; discussion 75-77.

32. Servadei F, Murray GD, Penny K, et al. The value of the "worst" computed tomographic scan in clinical studies of moderate and severe head injury. European Brain Injury Consortium. Neurosurgery 2000;46:70-75; discussion 75-77. 
33. Bullock MR, Chesnut R, Ghajar J, et al. Surgical management of traumatic parenchymal lesions. Neurosurgery 2006;58:S25-46; discussion $\mathrm{Si}-\mathrm{iv}$.

34. Meythaler JM, Peduzzi JD, Eleftheriou E, Novack TA. Current concepts: diffuse axonal injury-associated traumatic brain injury. Arch Phys Med Rehabil 2001;82:1461-1471.

35. Gentry LR, Godersky JC, Thompson B. MR imaging of head trauma: review of the distribution and radiopathologic features of traumatic lesions. AJR Am J Roentgenol 1988;150:663-672.

36. Scheid R, Ott DV, Roth H, Schroeter ML, von Cramon DY. Comparative magnetic resonance imaging at 1.5 and 3 Tesla for the evaluation of traumatic microbleeds. J Neurotrauma 2007;24:18111816.

37. Huisman TA, Sorensen AG, Hergan K, Gonzalez RG, Schaefer PW. Diffusion-weighted imaging for the evaluation of diffuse axonal injury in closed head injury. J Comput Assist Tomogr 2003;27:5-11.

38. Kinoshita T, Moritani T, Hiwatashi A, et al. Conspicuity of diffuse axonal injury lesions on diffusion-weighted MR imaging. Eur J Radiol 2005;56:5-11.

39. Marmarou A, Fatouros PP, Barzo P, et al. Contribution of edema and cerebral blood volume to traumatic brain swelling in headinjured patients. J Neurosurg 2000;93:183-193.

40. Langfitt TW, Tannanbaum HM, Kassell NF. The etiology of acute brain swelling following experimental head injury. J Neurosurg 1966;24:47-56.

41. Marmarou A, Signoretti S, Fatouros PP, Portella G, Aygok GA, Bullock MR. Predominance of cellular edema in traumatic brain swelling in patients with severe head injuries. J Neurosurg 2006;104:720-730.

42. Aldrich EF, Eisenberg HM, Saydjari C, et al. Diffuse brain swelling in severely head-injured children. A report from the NIH Traumatic Coma Data Bank. J Neurosurg 1992;76:450-454.

43. Polin RS, Shaffrey ME, Bogaev CA, et al. Decompressive bifrontal craniectomy in the treatment of severe refractory posttraumatic cerebral edema. Neurosurgery 1997;41:84-92; discussion 92-84.

44. Guerra WK, Gaab MR, Dietz H, Mueller JU, Piek J, Fritsch MJ. Surgical decompression for traumatic brain swelling: indications and results. J Neurosurg 1999;90:187-196.

45. Kunze E, Meixensberger J, Janka M, Sorensen N, Roosen K. Decompressive craniectomy in patients with uncontrollable intracranial hypertension. Acta Neurochir Suppl 1998;71:16-18.

46. Munch E, Horn P, Schurer L, Piepgras A, Paul T, Schmiedek P. Management of severe traumatic brain injury by decompressive craniectomy. Neurosurgery 2000;47:315-322; discussion 322-313.

47. Mirvis SE, Wolf AL, Numaguchi Y, Corradino G, Joslyn JN. Posttraumatic cerebral infarction diagnosed by $\mathrm{CT}$ : prevalence, origin, and outcome. AJR Am J Roentgenol 1990;154:1293-1298.

48. Pasqualin A, Vivenza C, Rosta L, Licata C, Cavazzani P, Da Pian R. Cerebral vasospasm after head injury. Neurosurgery 1984;15:855-858.

49. The Brain Trauma Foundation. The American Association of Neurological Surgeons. The Joint Section on Neurotrauma and
Critical Care. Computed tomography scan features. J Neurotrauma 2000;17:597-627.

50. Marshall LF, Marshall SB, Klauber MR, Clark MVB. A new classification of head injury based on computed tomography. J Neurosurg 1991;75:S14-S20.

51. Maas AI, Hukkelhoven CW, Marshall LF, Steyerberg EW. Prediction of outcome in traumatic brain injury with computed tomographic characteristics: a comparison between the computed tomographic classification and combinations of computed tomographic predictors. Neurosurgery 2005;57:1173-1182; discussion $1173-1182$

52. Wintermark M, van Melle G, Schnyder P, et al. Admission perfusion CT: prognostic value in patients with severe head trauma. Radiology 2004;232:211-220.

53. Arfanakis K, Haughton VM, Carew JD, Rogers BP, Dempsey RJ, Meyerand ME. Diffusion tensor MR imaging in diffuse axonal injury. AJNR Am J Neuroradiol 2002;23:794-802.

54. Niogi SN, Mukherjee P, Ghajar J, et al. Extent of microstructural white matter injury in postconcussive syndrome correlates with impaired cognitive reaction time: a $3 \mathrm{~T}$ diffusion tensor imaging study of mild traumatic brain injury. AJNR Am J Neuroradiol 2008;29:967-973

55. Huisman TA, Schwamm LH, Schaefer PW, et al. Diffusion tensor imaging as potential biomarker of white matter injury in diffuse axonal injury. AJNR Am J Neuroradiol 2004;25:370-376.

56. Rauscher A, Sedlacik J, Barth M, Mentzel HJ, Reichenbach JR. Magnetic susceptibility-weighted MR phase imaging of the human brain. AJNR Am J Neuroradiol 2005;26:736-742.

57. Sehgal V, Delproposto Z, Haacke EM, et al. Clinical applications of neuroimaging with susceptibility-weighted imaging. J Magn Reson Imaging 2005;22:439-450.

58. Tong KA, Ashwal S, Holshouser BA, et al. Hemorrhagic shearing lesions in children and adolescents with posttraumatic diffuse axonal injury: improved detection and initial results. Radiology 2003;227:332-339.

59. Akiyama Y, Miyata K, Harada K, et al. Susceptibility-weighted magnetic resonance imaging for the detection of cerebral microhemorrhage in patients with traumatic brain injury. Neurol Med Chir (Tokyo) 2009;49:97-99; discussion 99.

60. Tong KA, Ashwal S, Holshouser BA, et al. Diffuse axonal injury in children: clinical correlation with hemorrhagic lesions. Ann Neurol 2004;56:36-50.

61. Babikian T, Freier MC, Tong KA, et al. Susceptibility weighted imaging: neuropsychologic outcome and pediatric head injury. Pediatr Neurol 2005;33:184-194.

62. Huang M, Theilmann RJ, Robb A, et al. Integrated imaging approach with MEG and DTI to detect mild traumatic brain injury in military and civilian patients. J Neurotrauma 2009;26:1213-1226.

63. McAllister TW, Saykin AJ, Flashman LA, et al. Brain activation during working memory 1 month after mild traumatic brain injury: a functional MRI study. Neurology 1999;53:1300-1308. 